\title{
Pacific
}

Journal of

Mathematics

\section{BRAIDED GROUPS OF HOPF ALGEBRAS OBTAINED BY} TWISTING

\author{
D. GUREVICH AND SHAHN MAJID
}




\title{
BRAIDED GROUPS OF HOPF ALGEBRAS OBTAINED BY TWISTING
}

\author{
D. Gurevich and Shahn Majid
}

\begin{abstract}
It is known that every quasitriangular Hopf algebra $H$ can be converted by a process of transmutation into a braided group $B(H, H)$. The latter is a certain braided-cocommutative Hopf algebra in the braided monoidal category of $H$-modules. We use this transmutation construction to relate two approaches to the quantization of enveloping algebras.

Specifically, we compute $B(\widetilde{H}, \widetilde{H})$ in the case when $\widetilde{H}$ is the quasitriangular Hopf algebra (quantum group) obtained by Drinfeld's twisting construction on a cocommutative Hopf algebra $H$. In the case when $\widetilde{H}$ is triangular we recover the $S$-Hopf algebra $H_{F}$ previously obtained as a deformation-quantization of $H$. Here $H_{F}$ is a Hopf algebra in a symmetric monoidal category. We thereby extend the definition of $H_{F}$ to the braided case where $\widetilde{H}$ is strictly quasitriangular. We also compute its structure to lowest order in a quantization parameter $\hbar$. In this way we show that $B\left(U_{q}(g), U_{q}(g)\right)$ is the quantization of a certain generalized Poisson bracket associated to the Drinfeld-Jimbo solution of the classical Yang-Baxter equations.
\end{abstract}

1. Introduction. Hopf algebras in braided monoidal categories have been introduced in [10] and [11] in the context of Tannaka-Krein reconstruction theorems. It is shown there that every quantum group gives rise to a Hopf algebra in a braided category by a process of transmutation. The category is that of representations of the quantum group, and in this braided category the resulting Hopf algebra is in a certain sense "braided-cocommutative", i.e. like a group algebra. Hence such Hopf algebras in braided categories are called braided groups. The process is called transmutation because it turns a quantum group in the ordinary category of Hopf algebras into a group-like object in a non-commutative category.

Hopf algebras in symmetric monoidal categories, on the other hand, arise naturally in the deformation-quantization of triangular solutions of the classical Yang-Baxter equations (CYBE) [6]. They have been called $S$-Hopf algebras and are the enveloping algebras of $S$-Lie algebras and $S$-groups. Hence we are led to consider if braided groups, too, can arise as such deformation-quantization of some kind of Poisson structure. This is one motivation for the present paper. The 
answer generalizes the results of [6] to the braided case, as well as clarifying those results.

This deformation-quantization in [6] is achieved by means of an element $F$ constructed by Drinfeld in [1]. Let $g$ be a semisimple Lie algebra with universal enveloping algebra $g$, and $r \in \Lambda^{2} g$ a triangular solution of the CYBE. The corresponding $F$ lies in $(U(g) \otimes U(g))[[\hbar]]$ as a formal power-series in a parameter $\hbar$. The $S$-Hopf algebra in [6] is given on $C(G)$ (functions on the Lie group of $g$ ) with a multiplication deformed via $F$, and quantizing a certain Poisson bracket on $G$. In terms of $U(g)$ rather than $C(G)$, the $S$-Hopf algebra structure $U(g)_{F}$ is given by [6]

$$
\begin{aligned}
a \cdot{ }_{F} b & =\sum \operatorname{Ad}_{F^{(1)}}(a) \operatorname{Ad}_{F^{(2)}}(b), \\
\Delta_{F} & =\sum\left(\operatorname{Ad}_{F^{-(1)}} \otimes \operatorname{Ad}_{F^{-(2)}}\right) \circ \Delta,
\end{aligned}
$$

$$
S(a \otimes b)=\sum \operatorname{Ad}_{F^{-(1)} F^{(2)}}(b) \otimes \operatorname{Ad}_{F^{-(2)} F^{(1)}}(a)
$$

where $F=\sum F^{(1)} \otimes F^{(2)}$ and $F^{-1}=\sum F^{-(1)} \otimes F^{-(2)}$. The map $S: U(g)_{F} \otimes U(g)_{F} \rightarrow U(g)_{F} \otimes U(g)_{F}$ obeys the quantum Yang-Baxter equations $S_{12} S_{23} S_{12}=S_{23} S_{12} S_{23}$ and $S^{2}=\mathrm{id}$. The Poisson bracket in terms of $U(g)$ (rather than $C(G)$ ) defines a coPoisson structure on the vector space of $U(g)$. It is the map $\delta: U(g) \rightarrow U(g) \otimes U(g)$ defined by

$$
\delta a=-\sum\left[r^{(1)}, a_{(1)}\right] \otimes\left[r^{(2)}, a_{(2)}\right]
$$

where $r=\sum r^{(1)} \otimes r^{(2)}$ and $\Delta a=\sum a_{(1)} \otimes a_{(2)}$ in the Sweedler notation [13]. In fact, these formulae (1) can be used quite generally and define an $S$-Hopf algebra $H_{F}$ for any pair $(H, F)$ where $H$ is a cocommutative Hopf algebra and $F$ obeys a cocycle condition. The map $\Delta_{F}: H_{F} \rightarrow H_{F} \otimes H_{F}$ is an algebra homomorphism provided $H_{F} \otimes H_{F}$ has the algebra structure $(a \otimes b)(c \otimes d)=a S(b \otimes c) d$. Here we mean to first apply $S$ to $b \otimes c$ and then multiply the result on the left by $a$ and on the right by $d$. Thus $H_{F}$ is not an ordinary Hopf algebra.

This construction represents one approach to quantization. On the other hand, let us note that Drinfeld himself has used this same data $(H, F)$ quite differently to obtain a quantum group $\widetilde{H}$ or, more gemerally, a quasi-quantum group [3]. This represents a second (and more familiar) approach to quantization along the lines pioneered in [2] and [3]. The comultiplication of $\widetilde{H}$ is constructed by conjugating the comultiplication of $H$ by $F \in H \otimes H$. The multiplication of $H$ is not 
changed. In the case $H=U(g)$, this $\widetilde{U}(g)$ is thus a quantization of $U(g)$ as an ordinary (triangular) quantum group or Hopf algebra. It quantizes the solution $r$ (from which $F$ is obtained) viewed as a Poisson bracket on $C(G)$ in the sense of [2]. In the form of a coPoisson structure on $U(g)$ it is

$$
\delta a=-[r, \Delta a] .
$$

This is in contrast to (3).

Since the process of transmutation converts any quantum group into a braided group, and any triangular quantum group into one in a symmetric monoidal category, we can hope that these two quantizations, $\widetilde{U}(g)$ and $U(g)_{F}$, are connected by transmutation. In fact, we will show this quite generally, $B(\widetilde{H}, \widetilde{H}) \cong H_{F}$, where $H$ is cocommutative and $B($,$) denotes the braided-group transmutation con-$ struction of [10] and [11], albeit applied in the symmetric monoidal (unbraided) case. This is the main result of $\S 2$. This also clarifies the structure of $H_{F}$ itself. Note that the transmutation construction $B($,$) is a bifunctor, depending contravariantly in the first argument$ and covariantly in the second [10] [11]. This is the reason behind the notation.

Next, in $\S 3$, we generalize the results of $\S 2$ to the case where $H$ is viewed as a quasitriangular quasi-Hopf algebra $(H, \Phi, \mathscr{R})[3] . F$ is a cocycle with respect to this $\Phi$ and $\widetilde{H}$ is quasitriangular rather than triangular. This means that $B(\widetilde{H}, \widetilde{H})$ is strictly braided in the sense of [10] and [11] rather than an $S$-Hopf algebra in the sense of [5]. We therefore use the structure of $B(\widetilde{H}, \widetilde{H})$ and a suitable map $\alpha: H \rightarrow$ $B(\widetilde{H}, \widetilde{H})$ to define on the vector space of $H$ a generalization of $H_{F}$. The resulting deformation explicitly involves $\Phi$ and we denote it by $H_{F, \Phi}$.

These more general results of $\S 3$ are needed to deal with the important case $H=U(g, t)$, the quasitriangular quasi-Hopf algebra associated to a symmetric ad-invariant element $t \in g \otimes g$ [3]. Its multiplication and comultiplication coincide with those of $U(g)$. The necessary $\Phi$ was obtained in [3] by solving the Knizhnik-Zamolodchikov equations, and $\widetilde{H}$ is isomorphic to the Drinfeld-Jimbo quantum group $U_{q}(g)$. Our results therefore give an interpretation of the braided group $B\left(U_{q}(g), U_{q}(g)\right)$ as a deformation-quantization of the form $U(g, t)_{F, \Phi}$. We conclude the paper by computing its semiclassical structure. This takes the form of a certain Poisson-type structure on $g$ generalizing the one in (3) above, to the case where $r$ is strictly quasitriangular. 
2. $H_{F}$ obtained as a braided group $B(\widetilde{H}, \widetilde{H})$. We work throughout this section and the next with $H$ a Hopf algebra over a ring $k$. We use the standard notations, including the Sweedler formal sum notation for the comultiplication [13], $\Delta h=\sum h_{(1)} \otimes h_{(2)}$. The antipode is denoted $s: H \rightarrow H$ and the counit $\varepsilon: H \rightarrow k$. The axioms of a quasitriangular structure $\mathscr{R} \in H \otimes H$ are from [2]. They are such as to ensure that the category $H^{\mathscr{M}}$ of $H$-modules is a braided monoidal or "quasitensor" category. Here if $V, W$ are two $H$-modules of a quasitriangular Hopf algebra $(H, \mathscr{R})$, then the action of $h \in H$ on the tensor product module $V \otimes W$, and the quasi-symmetry or braiding isomorphisms $\Psi_{V, W}: V \otimes W \rightarrow W \otimes V$ are given by

$$
\begin{aligned}
h \triangleright(v \otimes w) & =\sum h_{(1)} \triangleright v \otimes h_{(2)} \triangleright w, \\
\Psi_{V, W}(v \otimes w) & =\sum \mathscr{R}^{(2)} \triangleright w \otimes \mathscr{R}^{(1)} \triangleright v
\end{aligned}
$$

where $\triangleright$ denotes the action of $H$. The functorial isomorphisms $\Psi$ play the role of "transposition" in the category. Finally, $H$ is triangular (rather than quasitriangular) if $\mathscr{R}_{21} \mathscr{R}=1 \otimes 1$. In this case the category $H^{\mathscr{M}}$ is an ordinary symmetric monoidal one. See [8, $\left.\S 7\right]$ for a systematic treatment of these well-known facts. We note that braided monoidal categories themselves were formally introduced into category theory in [7].

Next, we recall the construction of braided groups from [10] and [11]. We use the form in [11]. Let $(H, \mathscr{R})$ be any quasitriangular Hopf algebra. Let $B$ denote the linear space of $H$ viewed as an object in $H^{\mathscr{M}}$ by the Hopf algebra adjoint action $h \triangleright b=\sum h_{(1)} b s h_{(2)}$. We put on $B$ the same algebra structure and counit as $H$, and put a modified comultiplication and antipode according to [11],

$$
\begin{aligned}
\underline{\Delta} b & =\sum b_{(1)} s \mathscr{R}^{(2)} \otimes \mathscr{R}^{(1)} \triangleright b_{(2)}, \\
\underline{s} b & =\sum \mathscr{R}^{(2)} s\left(\mathscr{R}^{(1)} \triangleright b\right), \quad b \in B .
\end{aligned}
$$

The right-hand side is in terms of $H$. With this structure $B=$ $B(H, H)$ becomes a Hopf algebra in the braided monoidal category $H^{\mathscr{M}}[\mathbf{1 1}]$. The axioms for this are just as for ordinary Hopf algebras except that all the structure maps are now morphisms in the category (i.e. in our case $H$-equivariant), and $\Delta: B \rightarrow B \otimes B$ is an algebra homomorphism with respect to the braided tensor multiplication algebra structure of $B \otimes B$. This is defined with $\Psi_{B, B}$ in the role of 
the usual transposition. Such notions are well known in the symmetric monoidal case (see for example [12]) but work equally well when $\Psi^{2} \neq$ id. Finally, $B(H, H)$ is braided-cocommutative in a certain sense [11]. The construction of $B(H, H)$ that we have described is the diagonal case of a more general construction $B(),[11]$ yielding more general Hopf algebras in braided monoidal categories (such as braided-quasitriangular ones).

Our goal is to compute $B(\widetilde{H}, \widetilde{H})$ for certain quasitriangular Hopf algebras $\widetilde{H}$ obtained by the twisting construction of Drinfeld [3]. We now recall this in the special case that we need. For any Hopf algebra $H$ we say that an invertible $F \in H \otimes H$ is a "cocycle" if

$$
((\Delta \otimes \mathrm{id}) F) F_{12}=((\mathrm{id} \otimes \Delta) F) F_{23}
$$

where $F_{23}=1 \otimes F$ and $F_{12}=F \otimes 1$ in $H \otimes H \otimes H$.

Proposition 2.1 (cf. [3]). Let $(H, \mathscr{R})$ be a quasitriangular Hopf algebra and $F$ a cocycle. Then there is a new quasitriangular Hopf algebra $(\widetilde{H}, \widetilde{\mathscr{R}})$ defined by the same multiplication and counit and

$$
\widetilde{\Delta} h=F^{-1}(\Delta h) F, \quad \widetilde{\mathscr{R}}=F_{21}^{-1} \mathscr{R} F, \quad \tilde{s} h=u(s h) u^{-1}, \quad h \in \widetilde{H},
$$

where $u=\sum F^{-(1)}\left(s F^{-(2)}\right)$ and $u^{-1}=\sum\left(s F^{(1)}\right) F^{(2)}$.

Proof. This can easily be proven by direct computation, but in fact it is nothing other than a special case of Drinfeld's theory of twisting of quasi-Hopf algebras [3]. For this reason we defer the proof to $\S 3$, where it is a special case $(\Phi=1)$ of Lemma 3.1 and Proposition 3.2. The notation used in defining $u$ and $u^{-1}$ is $F=\sum F^{(1)} \otimes F^{(2)}$ and $F^{-1}=\sum F^{-(1)} \otimes F^{-(2)}$. We will use such explicit notations throughout.

LEMMA 2.2. In the setting of Proposition 2.1, the element $u^{-1}=$ $\sum\left(s F^{(1)}\right) F^{(2)}$ obeys

$$
\Delta u^{-1}=\left[(s \otimes s)\left(F_{21}^{-1}\right)\right]\left(u^{-1} \otimes u^{-1}\right) F^{-1}
$$


Proof. We compute

$$
\begin{aligned}
& \Delta u^{-1}=\sum\left(s F^{(1)}{ }_{(2)}\right) F^{(2)}{ }_{(1)} \otimes\left(s F^{(1)}{ }_{(1)}\right) F^{(2)}{ }_{(2)} \\
& =\sum s\left(F_{(2)}^{(1)} F_{(2)}^{\prime(1)}\right) F_{(3)}^{(1)} F^{\prime(2)} F^{-(1)} \\
& \otimes\left(s\left(F_{(1)}^{(1)} F_{(1)}^{(1)}\right)\right) F^{(2)} F^{-(2)} \\
& =\sum\left(s F^{\prime(1)}{ }_{(2)}\right) F^{\prime(2)} F^{-(1)} \otimes\left(s F_{(1)}^{\prime(1)}\right) u^{-1} F^{-(2)} \\
& =\sum\left(\left(s\left(F^{\prime(2)}{ }_{(1)} F^{(1)} F^{-(2)}\right)\right) F^{\prime(2)}{ }_{(2)} F^{(2)} \otimes\left(s\left(F^{\prime(1)} F^{-(1)}\right)\right) u^{-1}\right) F^{-1} \\
& =\sum\left(\left(s F^{-(2)}\right) u^{-1} \otimes\left(s F^{-(1)}\right) u^{-1}\right) F^{-1} \text {. }
\end{aligned}
$$

Here $F^{\prime}$ is another copy of $F$. The second and fourth equalities use the cocycle condition (7).

We are interested in computing $B=B(\widetilde{H}, \widetilde{H})$ in terms of $H$. For the remainder of the section we assume that the initial $H$ is cocommutative and $\mathscr{R}=1 \otimes 1$. In this case, $\widetilde{H}$ is triangular because, evidently, $\widetilde{\mathscr{R}}=F_{21}^{-1} F$ obeys $\widetilde{\mathscr{R}}_{21} \widetilde{\mathscr{R}}=1 \otimes 1$. In fact, Lemmas 2.4 and 2.5 hold more generally for any $(H, \mathscr{R})$. The other results hold more generally only after including the quasitriangular structure of $H$ in the formulae. We focus on the simplest and most important case when $H$ is cocommutative.

In this case, $H$ itself is a Hopf algebra in the category $H^{\mathscr{M}}$ by the adjoint action, while $B$ lives in the category $\widetilde{H}^{\mathscr{M}}$ and not in $H^{\mathscr{M}}$. However, the process of twisting is such that these categories are necessarily equivalent [3]. In our case, this simply comes out as

Proposition 2.3. Let $H$ be cocommutative and $F$ a cocycle. Then the symmetric monoidal category $\widetilde{H}^{\mathscr{M}}$ can be identified with the category ${ }_{H} \mathscr{M}_{F}$ of $H$-modules equipped with a deformed monoidal structure and symmetry

$$
\begin{aligned}
h \triangleright\left(v \otimes_{F} w\right) & =\sum F^{-(1)} h_{(1)} F^{(1)} \triangleright v \otimes_{F} F^{-(2)} h_{(2)} F^{(2)} \triangleright w, \\
\Psi_{V, W}\left(v \otimes_{F} w\right) & =\sum F^{-(1)} F^{(2)} \triangleright w \otimes_{F} F^{-(2)} F^{(1)} \triangleright v
\end{aligned}
$$

where $\triangleright$ denotes the relevant action of $H$.

Proof. This is immediate since the twisting in Proposition 2.1 does not change the algebra structure, so $\widetilde{H}=H$ as an algebra. Note that there is still a tensor functor (id, $c):{ }_{H} \mathscr{M} \rightarrow{ }_{H} \mathscr{M}_{F}=\widetilde{H}^{\mathscr{M}}$ given by the linear identity on objects and morphisms, but a non-trivial 
isomorphism of the tensor product of objects: $c_{V, W}: V \otimes W \cong V \otimes_{F} W$ as $H$-modules, given by the action of $F$.

LEMMA 2.4. Let $H_{F}$ denote the vector space of $H$ viewed in ${ }_{H} \mathscr{M}_{F}$ by equipping it with the action Ad of $H$

$$
\operatorname{Ad}_{h}(a)=\sum h_{(1)} a s h_{(2)}, \quad a, h \in H
$$

We identify this Ad as an action of $\widetilde{H}$ also. We denote by $\widetilde{\mathrm{Ad}}$ the "quantum" adjoint action of $\widetilde{H}$ on itself,

$$
\widetilde{\operatorname{Ad}}_{h}(b)=\sum h_{(\tilde{1})} b \tilde{s} h_{(\tilde{2})}, \quad h, b \in \widetilde{H} .
$$

Then there is an isomorphism $\alpha: H_{F} \rightarrow \widetilde{H}$ of $\widetilde{H}$-modules given by

$$
\alpha(a)=\sum\left(\operatorname{Ad}_{F^{(1)}} a\right) F^{(2)} .
$$

Proof. We first compute, another useful form of $\alpha$ as

$$
\begin{aligned}
\alpha(a) & =\sum F_{(1)}^{(1)} a\left(s F^{(1)}{ }_{(2)}\right) F^{(2)} \\
& =\sum F^{(1)} F^{-(1)} a\left(s\left(F^{(2)}{ }_{(1)} F^{(1)} F^{-(2)}\right)\right) F_{(2)}^{(2)} F^{\prime(2)} \\
& =\sum F^{-(1)} a\left(s F^{-(2)}\right) u^{-1}
\end{aligned}
$$

Here the second equality was from (7). This form (10) of $\alpha$ then implies that $\alpha$ is invertible with $\alpha^{-1}(a)=\sum F^{(1)} a u s F^{(2)}$, and

$$
\begin{aligned}
\widetilde{\operatorname{Ad}}_{h} \alpha(a) & =\sum h_{(\tilde{1})} \alpha(a) \tilde{s} h_{(\tilde{2})} \\
& =\sum F^{-(1)} h_{(1)} F^{(1)} \alpha(a) u\left(s\left(F^{-(2)} h_{(2)} F^{(2)}\right)\right) u^{-1} \\
& =\sum F^{-(1)} h_{(1)} F^{(1)} \alpha(a) u\left(s F^{(2)}\right)\left(s h_{(2)}\right)\left(s F^{-(2)}\right) u^{-1} \\
& =\alpha\left(\operatorname{Ad}_{h} a\right)
\end{aligned}
$$

as required.

LEMMA 2.5. The map $\alpha: H_{F} \rightarrow \widetilde{H}$ in Lemma 2.4 is an algebra homomorphism. Here $\widetilde{H}=H$ as an algebra and $H_{F}$ has the multiplication $\cdot F$ as shown in (1). 
Proof. We compute

$$
\begin{aligned}
\alpha\left(a \cdot_{F} b\right) & =\sum \alpha\left(\left(\operatorname{Ad}_{F^{(1)}} a\right)\left(\operatorname{Ad}_{F^{(2)}} b\right)\right) \\
& =\sum\left[\operatorname{Ad}_{F^{\prime(1)}}\left(\left(\operatorname{Ad}_{F^{(1)}} a\right)\left(\operatorname{Ad}_{F^{(2)}} b\right)\right)\right] F^{\prime(2)} \\
& =\sum\left(\operatorname{Ad}_{F_{(1)}^{(1)} F^{(1)}} a\right)\left(\operatorname{Ad}_{F_{(2)}^{\prime(1)} F^{(2)}} b\right) F^{\prime(2)} \\
& =\sum\left(\operatorname{Ad}_{F^{(1)}} a\right)\left(\operatorname{Ad}_{\left.F_{(1)}^{(2)} F^{(1)} b\right) F_{(2)}^{(2)} F^{(2)}}\right. \\
& =\sum\left(\operatorname{Ad}_{F^{(1)}} a\right) F_{(1)}^{(2)}\left(\operatorname{Ad}_{F^{\prime(1)}} b\right)\left(s F_{(2)}^{(2)}\right) F_{(3)}^{(2)} F^{\prime(2)} \\
& =\alpha(a) \alpha(b)
\end{aligned}
$$

as required. Here the second equality uses the definition of $\alpha$ from Lemma 2.4, the third the fact that the adjoint action of $H$ on $H$ respects its multiplication. The fourth equality is the cocycle condition. We then evaluate the adjoint action for the action of $F^{(2)}{ }_{(1)}$ and use the antipode axioms.

LeMmA 2.6. Let $H$ be cocommutative and $F$ a cocycle. Then the map $\alpha: H_{F} \rightarrow B(\widetilde{H}, \widetilde{H})$ in Lemma 2.4 is a coalgebra morphism. Here $B(\widetilde{H}, \widetilde{H})$ coincides with $\widetilde{H}$ as an algebra and an $\widetilde{H}$-module, but equipped with the braided comultiplication $\triangleq . H_{F}$ is equipped with the comultiplication $\Delta_{F}$ as shown in (1).

Proof. We first compute the expression $\Delta \alpha(a)$ needed in (6) for $\underline{\Delta} \alpha a$. Using $\alpha$ in the form (10) we have

$$
\begin{aligned}
& \Delta \alpha(a)= \sum F_{(1)}^{-(1)} a_{(1)}\left(s F^{-(2)}{ }_{(2)}\right)\left(u^{-1}\right)_{(1)} \\
& \otimes F^{-(1)}{ }_{(2)} a_{(2)}\left(s F^{-(2)}{ }_{(1)}\right)\left(u^{-1}\right)_{(2)} \\
&=\left(\sum F^{-(1)}{ }_{(1)} a_{(1)} s\left(F^{-(2)} F^{-(2)}{ }_{(2)}\right)\right. \\
&\left.\otimes F^{-(1)}{ }_{(2)} a_{(2)} s\left(F^{\prime-(1)} F^{-(2)}{ }_{(1)}\right)\right)\left(u^{-1} \otimes u^{-1}\right) F^{-1} \\
&=\left(\sum F_{(1)}^{-(1)} F_{(1)}^{\prime-(1)} a_{(1)} s F^{-(2)}\right. \\
&\left.\otimes F^{-(1)}{ }_{(2)} F^{-(1)}{ }_{(2)} a_{(2)} s\left(F^{-(2)} F^{\prime-(1)}{ }_{(3)}\right)\right)\left(u^{-1} \otimes u^{-1}\right) F^{-1} .
\end{aligned}
$$

The second equality uses Lemma 2.2 and the third the cocycle condition (7). Next, for $\underline{\Delta}$ in (6) we use an equivalent expression $\underline{\Delta} b=$ 
$\sum b_{(1)} \mathscr{R}^{(2)} S \mathscr{R}^{\prime(2)} \otimes \mathscr{R}^{\prime(1)} b_{(2)} \mathscr{R}^{(1)}[11]$, now applied to $(\widetilde{H}, \widetilde{\mathscr{R}})$. Then

$$
\begin{aligned}
& \underline{\Delta} \alpha(a)=\sum F^{-(1)} \alpha(a)_{(1)} F^{(1)} \widetilde{\mathscr{R}}^{(2)} u\left(s \widetilde{\mathscr{R}}^{\prime(2)}\right) u^{-1} \\
& \otimes \widetilde{\mathscr{R}}^{\prime(1)} F^{-(2)} \alpha(a)_{(2)} F^{(2)} \widetilde{\mathscr{R}}^{(1)} \\
& =\sum F^{-(1)} \alpha(a)_{(1)} F^{(2)} u\left(s F^{\prime(2)}\right)\left(s F^{\prime-(1)}\right) u^{-1} \\
& \otimes F^{\prime-(2)} F^{\prime(1)} F^{-(2)} \alpha(a)_{(2)} F^{(1)} \\
& =\sum F^{-(1)} \alpha(a)_{(2)} F^{(2)} u\left(s F^{\prime(2)}\right)\left(s F^{\prime-(1)}\right) u^{-1} \\
& \otimes F^{\prime-(2)} F^{\prime(1)} F^{-(2)} \alpha(a)_{(1)} F^{(1)} \\
& =\sum F^{-(1)} F_{(2)}^{\prime-(1)}\left(\operatorname{Ad}_{F^{\prime \prime(1)}(2)} a_{(2)}\right)\left(s F^{\prime-(2)}\right)\left(s F^{(2)}\right)\left(s F^{\prime \prime \prime-(1)}\right) u^{-1} \\
& \otimes F^{\prime \prime \prime-(2)} F^{(1)} F^{-(2)} F^{\prime-(1)}{ }_{(1)} F^{\prime \prime-(1)}{ }_{(1)} a_{(1)}\left(s F^{\prime \prime-(2)}\right) u^{-1} \\
& =\sum F^{-(1)} F^{\prime-(1)}\left(\operatorname{Ad}_{F^{\prime \prime(1)}} a_{(1)}\right)\left(s\left(F^{(2)} F^{\prime-(2)}\right)\right)\left(s F^{\prime \prime \prime-(1)}\right) u^{-1} \\
& \otimes F^{\prime \prime \prime-(2)} F^{(1)} F^{-(2)} F^{\prime-(1)}{ }_{(2)} F^{\prime \prime-(1)}{ }_{(2)} a_{(2)}\left(s F^{\prime \prime-(2)}\right) u^{-1} \\
& =\sum F^{\prime-(1)}\left(\operatorname{Ad}_{F^{\prime \prime-(1)}(1)} a_{(1)}\right)\left(s F^{\prime-(2)}(1)\right)\left(s F^{-(1)}\right) u^{-1} \\
& \otimes F^{-(2)} F^{\prime-(2)}{ }_{(2)} F^{\prime \prime-(1)} a_{(2)}\left(s F^{\prime \prime-(2)}\right) u^{-1} \\
& =\sum F^{\prime-(1)} F^{-(1)}{ }_{(1)}\left(\operatorname{Ad}_{F^{\prime-(1)}(1)} a_{(1)}\right)\left(s F^{-(1)}{ }_{(2)}\right)\left(s F^{\prime-(2)}\right) u^{-1} \\
& \otimes F^{-(2)} F^{\prime \prime-(1)}(2) a_{(2)}\left(s F^{\prime \prime-(2)}\right) u^{-1} \\
& =\sum F^{\prime-(1)}\left(\operatorname{Ad}_{F^{-(1)} F_{(1)}^{\prime-(1)}} a_{(1)}\right)\left(s F^{\prime-(2)}\right) u^{-1} \\
& \otimes F^{-(2)} F^{\prime \prime-(1)}{ }_{(2)} a_{(2)}\left(s F^{\prime \prime-(2)}\right) u^{-1} \\
& =\sum F^{\prime-(1)}\left(\operatorname{Ad}_{F^{\prime \prime-(1)}} a_{(1)}\right)\left(s F^{\prime-(2)}\right) u^{-1} \\
& \otimes F^{-(1)} F^{\prime \prime-(2)}{ }_{(1)} a_{(2)}\left(s F^{\prime \prime-(2)}{ }_{(2)}\right)\left(s F^{-(2)}\right) u^{-1} \\
& =(\alpha \otimes \alpha) \circ \Delta_{F}(a) \text {. }
\end{aligned}
$$

The third equality uses that $H$ is cocommutative, and the fourth uses the result for $\Delta \alpha(a)$ and the ensuing cancellations of $F^{-1} F$ and $u^{-1} u$. The fifth uses cocommutativity of $H$ again. The sixth, seventh and ninth equalities are each a use of the cocycle condition (7) and cocommutativity.

LEMMA 2.7. Let $H$ be cocommutative and $F$ a cocycle. Then the antipode $s_{F}$ on $H_{F}$ obtained via $\alpha$ from the antipode on $B(\widetilde{H}, \widetilde{H})$, coincides with the antipode $s$ on $H$. 
Proof. We compute $s_{F}=\alpha^{-1} \circ \underline{s} \circ \alpha$ from (6),

$$
\begin{aligned}
& s_{F}(a)=\sum F^{(1)} \widetilde{\mathscr{R}}^{(2)}\left(\tilde{s} \widetilde{\mathrm{Ad}} \widetilde{\mathscr{R}}^{(1)} \alpha(a)\right) u s F^{(2)} \\
& =\sum F^{(1)} \widetilde{\mathscr{R}}^{(2)} u\left(s \alpha\left(\operatorname{Ad}_{\widetilde{R}^{(1)}} a\right)\right) s F^{(2)} \\
& =\sum F^{(1)} F^{\prime-(1)} F^{\prime(2)} F^{-(1)}\left(s F^{-(2)}\right)\left(s F^{\prime \prime(2)}\right) \\
& \text { - }\left(s \operatorname{Ad}_{F^{\prime \prime(1)}} F^{\prime-(2)} F^{\prime(1)}(a)\right) s F^{(2)} \\
& =\sum F^{(1)} F^{\prime-(1)} F^{-(1)}(2) S\left(F^{\prime \prime(2)} F^{-(2)}\right) \\
& \text { - }\left(\operatorname{Ad}_{\left.F^{\prime \prime(1)} F^{\prime-(2)} F_{(1)}^{-(1)}(s a)\right) s F^{(2)}}\right. \\
& =\sum F^{(1)} F^{-(1)}\left(s F_{(2)}^{-(2)}\right)\left(\operatorname{Ad}_{F_{(1)}^{-(2)}}(s a)\right) s F^{(2)} \\
& =\sum F^{(1)} F^{-(1)}\left(s F^{-(2)}{ }_{(3)}\right) F^{-(2)}{ }_{(1)}(s a)\left(s F^{-(2)}{ }_{(2)}\right) s F^{(2)}=s(a)
\end{aligned}
$$

as required. For the second equality we used Lemma 2.6. For the third equality the definition of $\alpha$ and $u$. For the fourth equality we use $F_{12}^{\prime} F_{23}$ computed from the cocycle condition and then cancel one of the resulting $F$ 's using the antipode axiom. Note that $s$ commutes with Ad for $H$ cocommutative. For the fifth equality we use cocommutativity and the cocycle condition, and then cancel $F F^{-1}$. Finally, we write out Ad and use cocommutativity and the antipode axioms to obtain the result.

THEOREM 2.8. Let $H$ be cocommutative and $F$ a cocycle. Let $H_{F}$ denote $H$ with the $S$-Hopf algebra structure as shown in (1), viewed as an object in the category of $\widetilde{H}$-modules by the adjoint action Ad of $H$. In this category, there is an isomorphism of Hopf algebras

$$
\alpha: H_{F} \cong B(\widetilde{H}, \widetilde{H})
$$

and $S=\Psi_{H_{F}, H_{F}}$.

This shows that Drinfeld's quantization method, that turns a cocommutative Hopf algebra and cocycle $(H, F)$ into a triangular Hopf algebra, is converted by transmutation into the corresponding $S$-Hopf algebra in the approach of [6]. This also clarifies the structure of $H_{F}$.

Corollary 2.9. Let $H$ be cocommutative and $F$ a cocycle. Then the multiplication $\cdot_{F}: H_{F} \otimes_{F} H_{F} \rightarrow H_{F}$, comultiplication $\Delta_{F}: H_{H} \rightarrow$ $H_{F} \otimes_{F} H_{F}$, antipode $S_{F}: H_{F} \rightarrow H_{F}$, counit $H_{F} \rightarrow k$ and unit $k \rightarrow H_{F}$ are all morphisms in the category ${ }_{H} \mathscr{M}_{F}$. Moreover, $H_{F}$ is cocommutative in the sense

$$
\Psi_{H_{F}, H_{F}} \circ \Delta_{F}=\Delta_{F}
$$


3. Generalization to $F$ a quasi-cocycle. In this section we generalise the results of $\S 2$ to the case when $F$ does not obey the cocycle equation but something weaker. This is needed to handle the familiar quantum groups $U_{q}(g)$ associated to a complex semisimple Lie algebra $g$. For this setting we need the data $(H, \Phi, \mathscr{R}, F)$ where $H$ is a Hopf algebra (typically, cocommutative) and at the same time $(H, \Phi, \mathscr{R})$ is a quasitriangular quasi-Hopf algebra.

Let us recall from [3] that a quasitriangular quasi-Hopf algebra means $(H, \Delta, \varepsilon, s, \alpha, \beta, \Phi, \mathscr{R})$ where first of all $H$ is a unital algebra and $\Delta: H \rightarrow H \otimes H$ an algebra homomorphism such that

$$
(\Delta \otimes \mathrm{id}) \circ \Delta=\Phi((\mathrm{id} \otimes \Delta) \circ \Delta()) \Phi^{-1} .
$$

The axioms for the counit $\varepsilon$ are as usual. The associativity element $\Phi \in H \otimes H \otimes H$ is inyertible and obeys (id $\otimes \varepsilon \otimes \mathrm{id}) \Phi=1$ and the pentagon cocycle condition

$$
\begin{aligned}
& (\mathrm{id} \otimes \mathrm{id} \otimes \Delta)(\Phi)(\Delta \otimes \mathrm{id} \otimes \mathrm{id})(\Phi) \\
& \quad=(1 \otimes \Phi)(\mathrm{id} \otimes \Delta \otimes \mathrm{id})(\Phi)(\Phi \otimes 1) .
\end{aligned}
$$

The invertible $\mathscr{R} \in H \otimes H$ still intertwines the comultiplication and its opposite, but the other two axioms of a quasitriangular structure are modified by $\Phi$, see [3]. We will not need their exact form. Finally, the antipode for a quasi-Hopf algebra consists of elements $\alpha, \beta \in H$ and $s: H \rightarrow H$ obeying

$$
\begin{aligned}
& \sum\left(s h_{(1)}\right) \alpha h_{(2)}=\varepsilon(h) \alpha, \quad \sum h_{(1)} \beta s h_{(2)}=\varepsilon(h) \beta, \quad \forall h \in H \\
& \sum \Phi^{(1)} \beta\left(s \Phi^{(2)}\right) \alpha \Phi^{(3)}=1, \quad \sum\left(s \Phi^{-(1)}\right) \alpha \Phi^{-(2)} \beta s \Phi^{-(3)}=1
\end{aligned}
$$

and determined uniquely up to a transformation $\alpha \mapsto u \alpha, \beta \mapsto \beta u^{-1}$, $s h \mapsto u(s h) u^{-1}$, for any invertible $u \in H$ [3].

Note that if $H$ is also a Hopf algebra (with the same $\Delta$ ) then (11) implies that $\Phi$ is ad-invariant in the sense

$$
\begin{aligned}
& \sum \Phi^{(1)} h_{(1)} \otimes \Phi^{(2)} h_{(2)} \otimes \Phi^{(3)} h_{(3)} \\
& =\sum h_{(1)} \Phi^{(1)} \otimes h_{(2)} \Phi^{(2)} \otimes h_{(3)} \Phi^{(3)}, \quad \forall h \in H .
\end{aligned}
$$

Likewise, if $H$ is cocommutative, it must be that $\mathscr{R}$ is ad-invariant in the sense

$$
\sum \mathscr{R}^{(1)} h_{(1)} \otimes \mathscr{R}^{(2)} h_{(2)}=\sum h_{(1)} \mathscr{R}^{(1)} \otimes h_{(2)} \mathscr{R}^{(2)}, \quad \forall h \in H
$$


LEMMA 3.1. Let $H$ be a Hopf algebra and $\Phi$ an ad-invariant cocycle in the sense of (12) (15). We also suppose that $\Phi$ and $\sum \Phi^{(1)}\left(s \Phi^{(2)}\right) \Phi^{(3)}$ are invertible. Then $(H, \Phi)$ is a quasi-Hopf algebra with the same $\Delta, s$ and $\alpha=c^{-1}, \beta=1$, where $c=\sum \Phi^{(1)}\left(s \Phi^{(2)}\right) \Phi^{(3)}$ $=\sum\left(s \Phi^{-(1)}\right) \Phi^{-(2)}\left(s \Phi^{-(3)}\right)$ is central.

Proof. The only part we need to check is the antipode. Using adinvariance of $\Phi$ it is easy to see that $c=\sum \Phi^{(1)}\left(s \Phi^{(2)}\right) \Phi^{(3)}, c^{\prime}=$ $\sum\left(s \Phi^{-(1)}\right) \Phi^{-(2)} s \Phi^{-(3)}$ are central. From this it follows that we can take $\alpha=c^{-1}, \beta=1$ and $s$ to satisfy three of the four axioms of a quasi-Hopf algebra. A result in [3, Prop. 1.3] then implies that the fourth is also satisfied and $c=c^{\prime}$. This last step can also be computed directly from (12).

We suppose now that we are in the situation of the preceding lemma. If $F$ obeys the $\Phi$-cocycle condition

$$
\Phi((\Delta \otimes \mathrm{id}) F) F_{12}=((\mathrm{id} \otimes \Delta) F) F_{23}
$$

then $\widetilde{H}$ defined by twisting by $F$ is a Hopf algebra because $\widetilde{\Phi}=1$ [3]. If $\mathscr{R}$ makes $(H, \Phi)$ quasitriangular then $\widetilde{H}$ is quasitriangular.

Proposition 3.2. If $H$ is a Hopf algebra and $(H, \Phi, \mathscr{R})$ a quasitriangular quasi-Hopf algebra, and $F$ obeys the $\Phi$-cocycle condition, then the quasitriangular Hopf algebra $\widetilde{H}$ has the same multiplication and counit, and

$$
\begin{gathered}
\widetilde{\Delta}=F^{-1}(\Delta()) F, \quad \widetilde{\mathscr{R}}=F_{21}^{-1} \mathscr{R} F, \quad \tilde{s}=u(s()) u^{-1}, \\
u=\sum F^{-(1)} s F^{-(2)}
\end{gathered}
$$

where $u=\sum F^{-(1)}\left(s F^{-(2)}\right)$ and $u^{-1}=c^{-1} \sum\left(s F^{(1)}\right) F^{(2)}$.

Proof. We use the antipode for the quasi-Hopf algebra $(H, \Phi)$ from Lemma 3.1. After twisting using the formulae in [3] we have $\tilde{s}=$ $s, \tilde{\alpha}=c^{-1} \sum\left(s F^{(1)}\right) F^{(2)}$ and $\tilde{\beta}=\sum F^{-(1)}\left(s F^{-(2)}\right)$ and $\tilde{\alpha} \tilde{\beta}=\tilde{\beta} \tilde{\alpha}=1$ as $\widetilde{\Phi}=1$ in the antipode axioms applied to $\widetilde{H}$. We then make the transformation by $u$ to $\tilde{\alpha}=\tilde{\beta}=1$ and $\widetilde{S}=u(S()) u^{-1}$ and at the same time conclude the formula for $u^{-1}$.

Proposition 3.3. In the situation of Proposition 3.2, let $H_{F, \Phi}$ denote $H$ as an $\widetilde{H}$-module by the adjoint action of $H$. Here $\widetilde{H}$ coincides 
with $H$ as an algebra. Then the map $\alpha: H_{F, \Phi} \rightarrow \widetilde{H}$ defined by

$$
\begin{aligned}
\alpha(a) & =\sum F^{-(1)} a\left(s F^{-(2)}\right) u^{-1} \\
& =c^{-1} \sum \Phi^{(1)}\left(\operatorname{Ad}_{F^{(1)}}(a)\right)\left(s \Phi^{(2)}\right) \Phi^{(3)} F^{(2)}
\end{aligned}
$$

is an intertwiner, where $\widetilde{H}$ acts on $\widetilde{H}$ by the adjoint action of $\widetilde{H}$.

Proof. The proof in Lemma 2.4 that the first expression for $\alpha$ is an intertwiner goes through here without change. The computation that it equals the second expression is similar to that for Lemma 2.4, but with $F$ now a $\Phi$-cocycle.

Corollary 3.4. The morphism $\alpha$ in Proposition 3.3 induces on $H_{F, \Phi}$ a Hopf algebra structure in the braided monoidal category of $\widetilde{H}$-modules, where $\alpha\left(H_{F}, \Phi\right)=B(\widetilde{H}, \widetilde{H})$.

To see what the implied structure looks like explicitly, we compute the multiplication on $H_{F, \Phi}$ from the corollary, at least in the cocommutative case.

Proposition 3.5. Let $H$ be a cocommutative Hopf algebra, $\Phi$ an ad-invariant cocycle in the sense of (12) (15) and $F$ a $\Phi$-cocycle in the sense of (17). The multiplication on $H_{F, \Phi}$ implied by Corollary 3.4 takes the form

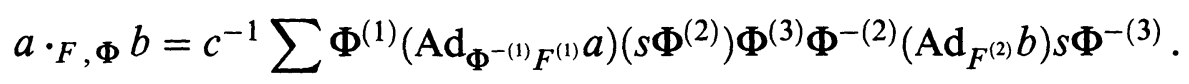

Proof. Let us first note that the element $X=\sum \Phi^{(1)} \otimes\left(s \Phi^{(2)}\right) \Phi^{(3)}$ in $H \otimes H$ is ad-invariant in the sense of (16), as follows from adinvariance of $\Phi$. Then the cocycle condition for $\Phi$ in (12), after applying $(\mathrm{id} \otimes \mathrm{id} \otimes \cdot)(\mathrm{id} \otimes \mathrm{id} \otimes S \otimes \mathrm{id})$ to both sides implies

$$
\begin{aligned}
& \sum \Phi^{(1)} \otimes X^{(1)} \Phi^{(2)}{ }_{(1)} \otimes\left(s \Phi^{(2)}{ }_{(2)}\right) X^{(2)} \Phi^{(3)} \\
& \quad=\sum X^{(1)}{ }_{(1)} \Phi^{-(1)} \otimes X^{(1)}{ }_{(2)} \Phi^{-(2)} \otimes\left(s \Phi^{-(3)}\right) X^{(2)}
\end{aligned}
$$


We now proceed to compute

$$
\begin{aligned}
& \alpha(a) \alpha(b)=c^{-2} \sum X^{(1)}\left(\operatorname{Ad}_{F^{(1)}} a\right) X^{(2)} F^{(2)} \Phi^{(1)}\left(\operatorname{Ad}_{F^{\prime(1)}} b\right)\left(s \Phi^{(2)}\right) \Phi^{(3)} F^{\prime(2)} \\
& =c^{-2} \sum X^{(1)}\left(\operatorname{Ad}_{F^{(1)}} a\right) X^{(2)} F_{(1)}^{(2)} \Phi^{(1)} \\
& \text { - }\left(\operatorname{Ad}_{F^{(1)}} b\right)\left(s \Phi^{(2)}\right)\left(s F^{(2)}{ }_{(2)}\right) F^{(2)}{ }_{(3)} \Phi^{(3)} F^{\prime(2)}
\end{aligned}
$$

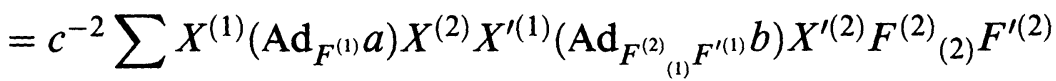

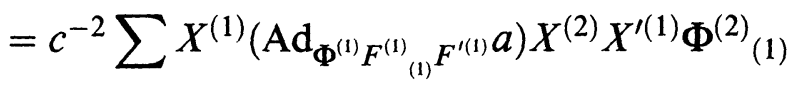

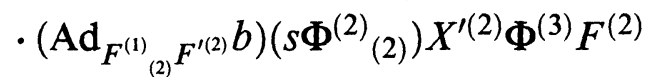

$$
\begin{aligned}
& =c^{-2} \sum X^{(1)} X^{\prime(1)}{ }_{(1)}\left(\operatorname{Ad}_{\Phi^{-(1)} F_{(1)}^{(1)} F^{\prime(1)}} a\right)\left(s X^{\prime(1)}{ }_{(2)}\right) X^{(2)}
\end{aligned}
$$

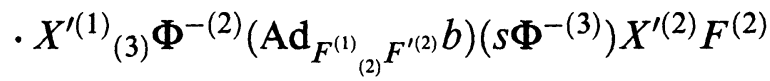

$$
\begin{aligned}
& =c^{-2} \sum X^{\prime(1)} X^{(1)}\left(\operatorname{Ad}_{\Phi^{-(1)} F_{(1)}^{(1)} F^{\prime(1)}} a\right) X^{(2)} \Phi^{-(2)}
\end{aligned}
$$

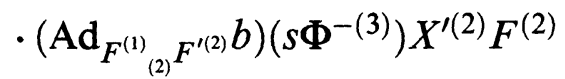

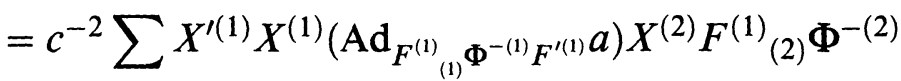

$$
\begin{aligned}
& \text { - }\left(\operatorname{Ad}_{F^{\prime(2)}} b\right)\left(s \Phi^{-(3)}\right)\left(s F_{(3)}^{(1)}\right) X^{\prime(2)} F^{(2)} \\
& =c^{-2} \sum X^{(1)} F_{(1)}^{(1)} X^{(1)}\left(\operatorname{Ad}_{\Phi^{-(1)} F^{\prime(1)}} a\right) X^{(2)} \Phi^{-(2)} \\
& \text { - }\left(\operatorname{Ad}_{F^{\prime(2)}} b\right)\left(s \Phi^{-(3)}\right)\left(s F_{(2)}^{(1)}\right) X^{\prime(2)} F^{(2)} \\
& =c^{-2} \sum X^{\prime(1)}\left(\operatorname { A d } _ { F ^ { ( 1 ) } } \left[X^{(1)}\left(\operatorname{Ad}_{\Phi^{-(1)} F^{(1)}} a\right) X^{(2)} \Phi^{-(2)}\right.\right. \\
& \text { - } \left.\left.\left(\operatorname{Ad}_{F^{\prime(2)}} b\right)\left(s \Phi^{-(3)}\right)\right]\right) X^{\prime(2)} F^{(2)} \\
& =\alpha\left(c^{-1} \sum X^{(1)}\left(\operatorname{Ad}_{\Phi^{-(1)} F^{\prime(1)}} a\right) X^{(2)} \Phi^{-(2)}\left(\operatorname{Ad}_{F^{\prime(2)}} b\right)\left(s \Phi^{-(3)}\right)\right)
\end{aligned}
$$

as required. For the third equality we used ad-invariance of $\Phi$ under $F^{(2)}$, followed by the definitions of $X^{\prime}$ (another copy of $X$ ) and Ad. For the fourth equality we used the $\Phi$-cocycle condition for $F$, and (18) above for the fifth equality. For the sixth equality we used adinvariance of $X$ under $X^{\prime(1)}{ }_{(1)}$ (relying on cocommutativity of $H$ ), and then cancelling. For the seventh equality we expanded $\operatorname{Ad}_{F^{(1)}}$ and used ad-invariance of $\Phi^{-(1)}$ under $F^{(1)}$. For the eighth equality. we likewise expanded $\operatorname{Ad}_{F_{(1)}^{(1)}}$ and used ad-invariance of $X$.

Thus the multiplication in $H_{F, \Phi}$ differs from the simpler form for $H_{F}$ in the preceding section by the inclusion of the element $\Phi$. This is needed in order to maintain associativity of the multiplication in our braided situation. It is clear that similar modifications for the 
comultiplication etc. are also needed, but that apart from including $\Phi$, the formulae are similar to those in $\S 2$.

4. Semiclassical limit of $B\left(U_{q}(g), U_{q}(g)\right)$. The quantum groups $U_{q}(g)$ are isomorphic to $\widetilde{H}$ where $H=U(g)$ in the setting of $\S 3$, and $\Phi, \mathscr{R}, F$ are given in [3]. $\mathscr{R}$ is given by $\mathscr{R}=e^{h t / 2}$ where $t$ is the split casimir. Hence $B\left(U_{q}(g), U_{q}(g)\right) \cong U(g)_{F, \Phi}$ where $U(g)_{F, \Phi}$ is defined by $\alpha$ in Corollary 3.4. This means that the braided groups of the Drinfeld-Jimbo quantum groups can be understood as deformation-quantizations of $U(g)$ in the form $U(g)_{F, \Phi}$. We conclude the paper with a brief look at their semiclassical structure, i.e., we compute their cocommutator $\delta$ to lowest order in the quantization parameter $\hbar$.

LEMMA 4.1. Let $U_{q}(g)$ be a quasitriangular Hopf algebra coinciding with $U(g)$ to $O(\hbar)$. Let $\mathscr{R}=1 \otimes 1+\hbar r+O\left(\hbar^{2}\right)$, where $r \in g \otimes g$. Let $U(g)_{F, \Phi}$ denote the braided Hopf algebra structure implied by Corollary 3.4, with comultiplication $\Delta_{F, \Phi}$. Then

$$
\begin{aligned}
\hbar^{-1}\left(\Delta_{F, \Phi}-\tau \circ \Delta_{F, \Phi}\right)= & \hbar^{-1}(\underline{\Delta}-\tau \circ \underline{\Delta}) \\
= & -\sum\left[r_{-}{ }^{(1)}, b_{(1)}\right] \otimes\left[r_{-}{ }^{(2)}, b_{(2)}\right] \\
& +\sum\left[r_{+}{ }^{(1)}, b_{(1)}\right] \otimes\left\{r_{+}{ }^{(2)}, b_{(2)}\right\}+O(\hbar)
\end{aligned}
$$

where $\tau$ is the usual twist map and $\{$,$\} denotes anticommutator.$

Proof. We write the map $\alpha=1+\hbar A+O\left(\hbar^{2}\right)$ and $\Delta=\Delta+\hbar D+O\left(\hbar^{2}\right)$, say. Hence $\Delta_{F, \Phi}=\Delta-\hbar(A \otimes \operatorname{id}) \Delta-\hbar(\operatorname{id} \otimes A) \Delta+\hbar \Delta \circ A+\hbar D+O\left(\hbar^{2}\right)$. Since $\Delta$ is cocommutative, the first result follows. It says that the semiclassical structure can be computed in $B\left(U_{q}(g), U_{q}(g)\right)$ as well as in $U(g)_{F, \Phi}$. Next, to compute the cocommutator we note that any braided group obtained by transmutation of a quantum group is necessarily braided-cocommutative in the sense [11]

$$
\sum \mathscr{R}^{(2)} Q^{(1)} \triangleright b_{\underline{(2)}} \otimes\left(\mathscr{R}^{(1)} \triangleright \underline{b}_{\underline{(1)}}\right) Q^{(2)}=\sum \underline{b}_{\underline{(1)}} \otimes b_{(\underline{2)}}
$$

where $\underline{\Delta} b=\sum b_{(1)} \otimes b_{(2)}$ and $Q=\mathscr{R}_{21} \mathscr{R}$. In our case, this immediately gives the linearized form shown. Here we used that the isomorphism $U_{q}(g) \cong \widetilde{U}(g)$ is also $1+O(\hbar)$ and that the quantum adjoint action differs from the usual one by order $\hbar$.

This is the semiclassical structure of the braided group $B\left(U_{q}(g), U_{q}(g)\right)$ or $U(g)_{F, \Phi}$ defined through it. Since the necessary quantum groups are obtained by quantizing a solution of the classical 
Yang-Baxter equation, we are motivated to verify the structure of $\delta$ directly in this context.

Proposition 4.2. Let $r$ be a solution of the classical Yang-Baxter equations, with $r=r_{-}+r_{+}$where $r_{-}$is antisymmetric and $r_{+}$is symmetric and ad-invariant. On the vector space of $U(g)$ we define a cobracket

$$
\delta b=-\sum\left[r_{-}{ }^{(1)}, b_{(1)}\right] \otimes\left[r_{-}{ }^{(2)}, b_{(2)}\right]+\sum\left[r_{+}{ }^{(1)}, b_{(1)}\right] \otimes\left\{r_{+}{ }^{(2)}, b_{(2)}\right\}
$$

where $\{$,$\} denotes anticommutator. This \delta$ is antisymmetric and a coderivation in each output. If $r_{+}=0$ it also obeys the coJacobi identity, so that in this case $\delta$ is a coPoisson structure.

Proof. Note that $\sum\left[r_{+}{ }^{(1)}, b_{(1)}\right] \otimes\left\{r_{+}{ }^{(2)}, b_{(2)}\right\}=-\sum\left\{r_{+}{ }^{(1)}, b_{(1)}\right\} \otimes$ $\left[r_{+}{ }^{(2)}, b_{(2)}\right]$ by ad-invariance of $r^{+}$(in the same sense as $(16)$ ), so that $\delta$ is manifestly antisymmetric. That it is a coderivation is equally easy. That it obeys the coJacobi identity requires a detailed computation and holds in general only if $r^{+}=0$.

We have not explicitly discussed the axioms of a coPoisson structure. However, they are just so that the dual space is a Poisson bracket algebra by dualizing $\delta$. See also [2]. If $r_{+}=0$ then we are in the setting of the Introduction and $\S 2$, and $\delta$ is indeed a coPoisson structure. But if $r_{+} \neq 0$ we are in the setting of $\S 3$ with an associativity cocycle $\Phi$. Hence it is not surprising in this case that $\delta$ does not generally obey the usual coJacobi identity. It should be viewed as some kind of generalized coPoisson structure. Likewise, in a topological context we have,

Corollary 4.3. Let $r \in g \otimes g$ be a solution of the CYBE on complex semisimple $g$, the Lie algebra of $G$. We suppose that $r=r_{-}+r_{+}$ where $r_{-}$is antisymmetric and $r_{+}$is symmetric and ad-invariant. Then $C(G)$ has the antisymmetric bracket structure

$$
\{a, b\}=-\sum \mathrm{ad}_{r_{-}(1)}^{*}(a) \operatorname{ad}_{r_{-}(2)}^{*}(b)+\sum \operatorname{ad}_{r_{+}(1)}^{*}(a)\left(L_{r_{+}(2)}^{*}+R_{r_{+}(2)}^{*}\right)(b) .
$$

It is a derivation in each input and if $r_{+}=0$, is a Poisson bracket.

Proof. This can be proven by formally dualizing the previous proposition or, since the result is in a slightly different context of Lie groups, directly. $L_{\xi}^{*}$ denotes the action of a Lie algebra element $\xi \in g$ by extension as a left-invariant vector field, and $R^{*}$ as a right-invariant 
vector field. The induced adjoint action is $\mathrm{ad}^{*}=L^{*}-R^{*}$. The case $r_{+}=0$ was observed in [6].

The actual deformation-quantization of $C(G)$ corresponding to this "generalized Poisson bracket" is given by the dual of the constructions above. Let $A=C(G)_{q}$ denote the quantum group of function algebra type dual to $U_{q}(g)$. These can be constructed as matrix quantum groups [4]. There is a braided group construction $B(A, A)$ for such dual quantum groups given in [10]. The generalized Poisson bracket above is nothing other than the lowest-order of $a \cdot b-b \cdot a$ for $:$ the multiplication in $B(A, A)$. Some examples, including $\mathrm{BSL}(2)=B\left(\mathrm{SL}_{q}(2), \mathrm{SL}_{q}(2)\right)$ were given in [9].

This generalized Poisson bracket in Corollary 4.3 is a precise generalization of the Poisson bracket on $C(G)$ for $r$ triangular [6], to the quasitriangular case. We note that whereas the Poisson bracket on $C(G)$ is highly degenerate in the triangular case, the presense of $r_{+}$ and the anticommutator makes it rather less degenerate in the strictly quasitriangular case. An axiomatic framework for this generalized Poisson structure is one direction for further work.

\section{REFERENCES}

[1] V. G. Drinfeld, On constant quasiclassical solutions of the Yang-Baxter equations, Soviet Math. Dokl., 28 (1983), 667-671.

[2] Quantum groups, in Proceedings of the ICM, A. Gleason, editor, Amer. Math. Soc., Providence, RI, 1987, pp. 798-820.

[3] _, Quasi-Hopf algebras, Eng. transl. in Leningrad Math. J., 1 (1990), 14191457, On quasitriangular quasi-Hopf algebras and a quantum group closely connected with $\mathrm{Gal}(\bar{Q} / Q)$, Eng. transl. in Leningrad Math. J., 2 (1991), 829.

[4] L. D. Faddeev, N. Yu. Reshetikhin, and L. A. Takhtajan, Quantization of Lie groups and Lie algebras, Eng. transl. in Leningrad Math. J., 1 (1990), 193-225.

[5] D. I. Gurevich, Algebraic aspects of the quantum Yang-Baxter equation, English transl. in Leningrad Math. J., 2 (1991), 801-828.

[6] D. I. Gurevich, V. Rubtsov, and N. Zobin, Quantization of Poisson pairs: the R-matrix approach, J. Geom. Phys., 9 (1992), 25-44.

[7] A. Joyal and R. Street, Braided monoidal categories, Math. Report 86008, Macquarie University, 1986.

[8] S. Majid, Quasitriangular Hopf algebras and Yang-Baxter equations, Internat. J. Modern Phys. A, 5 (1990), 1-91.

[9] _ Examples of braided groups and braided matrices, J. Math. Phys., 32 (1991), 3246-3253.

[10] _ Braided groups, 1990 to appear in J. Pure Appl. Algebra.

[11] __, Braided groups and algebraic quantum field theories, Lett. Math. Phys., 22 (1991), 167-176, Transmutation theory and rank for quantum braided groups, Math. Proc. Camb. Phil. Soc., 113 (1993), 45-70. 
[12] B. Pareigis, A non-commutative non-cocommutative Hopf algebra in nature, J. Algebra, 70 (1981), 356.

[13] M. E. Sweedler, Hopf Algebras, Benjamin, 1969.

Received January 2, 1992. The first author had a Fellowship Visit supported by the Royal Society, London. The second author was an SERC Research Fellow and Drapers Fellow of Pembroke College, Cambridge.

UNIVERSITY OF CAMBRIDGE

CAMBRIDGE CB3 9EW, UK 


\title{
PACIFIC JOURNAL OF MATHEMATICS
}

Founded by

\author{
E. F. BeCKenBACH (1906-1982) F. Wolf (1904-1989)
}

\section{EDITORS}

Sun-Yung A. Chang

(Managing Editor)

University of California

Los Angeles, CA 90024-1555

chang@math.ucla.edu

\section{F. Michael Christ}

University of California

Los Angeles, CA 90024-1555

christ@math.ucla.edu

Herbert Clemens

University of Utah

Salt Lake City, UT 84112

clemens@math.utah.edu
THOMAS ENRIGHT

University of California, San Diego

La Jolla, CA 92093

tenright@ucsd.edu

Nicholas ERCOLANI

University of Arizona

Tucson, AZ 85721

ercolani@math.arizona.edu

R. FINN

Stanford University

Stanford, CA 94305

finn@gauss.stanford.edu

VAUghan F. R. Jones

University of California

Berkeley, CA 94720

vfr@math.berkeley.edu
STEVEN KERCKHOFF

Stanford University

Stanford, CA 94305

spk@gauss.stanford.edu

Martin ScharLemanN University of California Santa Barbara, CA 93106 mgscharl@math.ucsb.edu

Harold Stark

University of California, San Diego La Jolla, CA 92093

V. S. VARADARAJAN University of California

Los Angeles, CA 90024-1555

vsv@math.ucla.edu

\section{SUPPORTING INSTITUTIONS}

UNIVERSITY OF ARIZONA

UNIVERSITY OF BRITISH COLUMBIA

CALIFORNIA INSTITUTE OF TECHNOLOGY

UNIVERSITY OF CALIFORNIA

UNIVERSITY OF MONTANA

UNIVERSITY OF NEVADA, RENO

NEW MEXICO STATE UNIVERSITY

OREGON STATE UNIVERSITY
UNIVERSITY OF OREGON

UNIVERSITY OF SOUTHERN CALIFORNIA

STANFORD UNIVERSITY

UNIVERSITY OF HAWAII

UNIVERSITY OF UTAH

WASHINGTON STATE UNIVERSITY

UNIVERSITY OF WASHINGTON 


\title{
PACIFIC JOURNAL OF MATHEMATICS
}

\author{
Volume $162 \quad$ No. $1 \quad$ January 1994
}

Semisimplicity of restricted enveloping algebras of Lie superalgebras $\quad 1$

JEFFERY MARC BERGEN

A classification of certain 3-dimensional conformally flat Euclidean hypersurfaces

OSCAR J. GARAY

Braided groups of Hopf algebras obtained by twisting

D. GUREVICH and SHAHN MAJID

Flat connections, geometric invariants and the symplectic nature of the 45 fundamental group of surfaces

K. GURUPRASAD

Spin models for link polynomials, strongly regular graphs and Jaeger's 57 Higman-Sims model

PIERRE DE LA HARPE

On the uniqueness of representational indices of derivations of $C^{*}$-algebras

EDWARD KISSIN

On infinitesimal behavior of the Kobayashi distance

MYUNG YULL PANG

Vertex operator construction of standard modules for $A_{n}^{(1)}$

MIRKO PRIMC

Bergman and Hardy spaces with small exponents

KEHE ZHU 